\title{
Activation of the nuclear factor E2-related factor 2/anitioxidant response element alleviates the nitroglycerin-induced hyperalgesia in rats
}

Wei $\mathrm{Di}^{1+}$, Xiaolei Shi ${ }^{2+}$, Hua $\mathrm{Lv}^{1}$, Jun Liu' ${ }^{1}$, Hong Zhang ${ }^{1}$, Zhiwei $\mathrm{Li}^{1}$ and Yannan Fang ${ }^{3^{*}}$

\begin{abstract}
Background: Antioxidants have been proven to weaken hyperalgesia in neuropathic pain. Endogenous antioxidant defense system may have a role in the prevention of hyperalgesia in migraine. In this study, we aimed to evaluate the role of nuclear factor E2-related factor 2/antioxidant response element (Nrf2/ARE) pathway in regulating the activation of the trigeminovascular system (TGVS) and hypersensitivity in nitroglycerin (NTG)-induced hyperalgesia rats.

Methods: The expression levels of $\mathrm{Nrf2}, \mathrm{HO}, \mathrm{HO}$, and $\mathrm{NQO} 1$ in the trigeminal nucleus caudalis (TNC) were detected by western blot. Immunofluorescence was used to demonstrate the cell-specific localization of Nrf2 in TNC. Sulforaphane, a Nrf2 activator, was administered to NTG-induced rats. Then, the number of c-Fos- and nNOS-immunoreactive neurons in TNC was evaluated using immunofluorescence, and c-Fos and nNOS protein levels were quantified using western blot. Von Frey hair testing was used to evaluate the tactile thresholds of rats at different time points in different groups.

Results: Total cellular and nuclear levels of the proteins Nrf2, HO1, and NQO1 were elevated in TNC after NTG injection, and Nrf2 was found to be located in the nucleus and cytoplasm of the neurons. Sulforaphane pretreatment significantly increased the nuclear Nrf2, HO1, and NQO1 levels in TNC. In addition, sulforaphane exposure effectively inhibited the expression of nNOS and c-Fos, reduced the number of nNOS and c-Fos immunoreactive neurons in TNC, and attenuated the tactile thresholds induced by NTG injection.

Conclusion: Oxidative stress was involved in nitroglycerin-induced hyperalgesia. Activation of the Nrf2/ARE pathway inhibited the activation of TGVS and prevented the induction of hyperalgesia. Sulforaphane might therefore be an effective agent for hyperalgesia. Further studies are needed to discover the underlying mechanisms of the process.
\end{abstract}

Keywords: Migraine, Nitroglycerin, Hyperalgesia, Nrf2/ARE, Trigeminovascular system

\footnotetext{
*Correspondence: yannanfang2012@qq.com

${ }^{\dagger}$ Equal contributors

${ }^{3}$ Guangdong Key Laboratory for Diagnosis and Treatment of Major

Neurological Diseases, Department of Neurology, National Key Clinical

Department and Key Discipline of Neurology, The First Affiliated Hospital,

Sun Yat-sen University, Guangdong 510080, China

Full list of author information is available at the end of the article
} 


\section{Background}

Migraine is a primary headache disorder characterized by recurring, episodic, and unilateral throbbing pain in the head. Cutaneous hyperalgesia occurs during migraine attacks, and is a risk factor for the recurrent attack and chronification of migraine [1, 2]. Exploring the potential mechanisms of this feature may warrant preventive treatment strategies.

Nitroglycerin (NTG) is widely used to create a validated animal model of migraine for further exploration of the pathogenesis and treatment of the disorder $[3,4]$. Systemic administration of NTG causes a delayed spontaneous headache attack and induces the consequent central sensitization in migraineurs or rats via an NOdependent pathway [5-7]. And trigeminovascular system (TGVS) activation participates in the nociceptive transmission, thereby enhancing this chronic sensitization process [8].

Several studies have shown that oxidative stress plays a role in central sensitization $[9,10]$. Reactive oxygen species scavenger alleviates hyperalgesia in rats with neuropathic pain, suggesting that potential role of antioxidants $[11,12]$. It has been found that the nuclear factor E2-related factor 2/antioxidant response element (Nrf2/ARE) pathway is the most important endogenous antioxidant defense system, and plays a critical role in regulating cellular oxidation, cell defense, and protection [13]. Increasing data points out the protective role of Nrf2/ARE pathway activation in the brain [14]. However, the role of Nrf2/ARE pathway in hyperalgesia in migraine remains unclear.

Thus, the aim of this study was to investigate the role of Nrf2/ARE pathway in NTG-induced hyperalgesia and its underlying mechanism. By doing so, we expect to find an effective therapeutic approach for this disorder.

\section{Methods \\ Animals}

Male Sprague-Dawley rats $(n=132$, weight $180-220 \mathrm{~g})$ obtained from the Laboratory Animal Center of Sun Yat-sen University (Guangzhou, China) were used for the study. The animals were housed in groups of 3-4 with water and food available ad libitum and were kept under a 12-h light/dark cycle at constant temperature $\left(25 \pm 1{ }^{\circ} \mathrm{C}\right)$ conditions. All experiments were conducted according to the international association for the study of pain (IASP) guideline and every effort was made to minimize animal suffering.

\section{Drug administration}

NTG (Beijing Yimin Pharmaceutical Co., Ltd, China) was injected subcutaneously (s.c) in the back of rats $(10 \mathrm{mg} / \mathrm{kg})$ from a stock of $5.0 \mathrm{mg} / \mathrm{ml}$. Control rats were subcutaneously injected with an equal volume of $0.9 \%$ normal saline (NS) as a vehicle for NTG [3].

R, S-Sulforaphane (SFN) (LKT Laboratories, Inc., St. Paul, MN) was dissolved in sterilized distilled water according to the instructions, and a dose of $5 \mathrm{mg} / \mathrm{kg}$ was administered intraperitoneally (i.p) based on previous studies $[15,16]$. The vehicle group was also injected intraperitoneally with an equal volume of sterilized water.

\section{Experimental protocol}

First, 60 rats were randomly separated into ten groups according to the different time points $(0,0.5 \mathrm{~h}, 1 \mathrm{~h}, 2 \mathrm{~h}$, and $4 \mathrm{~h}$ ) after NTG/NS injection. TNC tissue samples of the rats were taken for analyzing the expression levels of total cell Nrf2, nuclear Nrf2, HO, and NQO1 using western blot. A group of 18 rats was used to demonstrate the cell localization of Nrf2 in TNC among the groups (Control, NTG $2 \mathrm{~h}$, and NTG $4 \mathrm{~h}$ ) by immunofluorescence. Second, rats were divided into five groups as follows: 1$)$ Control group $(n=6)$, rats received NS (s.c) in a volume equal to that of NTG, 2) SFN plus control group $(n=6)$, rats received SFN (5 $\mathrm{mg} / \mathrm{kg}$ i.p) $30 \mathrm{~min}$ before NS (s.c), 3) NTG group $(n=6)$, rats received NTG (10 mg/kg s.c), 4) $\mathrm{H}_{2} \mathrm{O}$ plus NTG group $(n=6)$, rats received sterilized distilled water (i.p) $30 \mathrm{~min}$ before NTG (10 mg/kg s.c), and 5) SFN plus NTG group $(n=6)$, rats received SFN (5 mg/kg i.p) $30 \mathrm{~min}$ before NTG (10 mg/ $\mathrm{kg}, \mathrm{s.c}$ ). Von Frey hair testing was used to evaluate the tactile sensitivity threshold. Western blot was used to detect the c-Fos, nNOS, nuclear Nrf2, HO1, and NQO1 expressions in TNC. Finally, rats were divided into four groups as follows: 1$)$ Control group $(n=6)$, rats received a subcutaneous injection of NS (s.c) in a volume equal to that of NTG, 2) NTG group $(n=6)$, rats received NTG (10 mg/kg s.c), 3) $\mathrm{H}_{2} \mathrm{O}$ plus NTG group $(n=6)$, rats received sterilized distilled water (i.p) $30 \mathrm{~min}$ before NTG (10 mg/kg, s.c), and 4) SFN plus NTG group $(n=6)$, rats received SFN (5 mg/kg i.p) $30 \mathrm{~min}$ before NTG (10 $\mathrm{mg} / \mathrm{kg}$, s.c). Immunofluorescence was performed to evaluate the numbers of c-Fos and nNOS-immunoreactive neurons in TNC.

\section{Behavior test}

Tactile sensitivity threshold was evaluated with calibrated $(0.008$ g, 0.02 g, 0.04 g, 0.07 g, 0.4 g, 0.6 g, 1.0 g, $1.4 \mathrm{~g}, 2.0 \mathrm{~g}, 4.0 \mathrm{~g}, 6.0 \mathrm{~g}, 8.0 \mathrm{~g}, 10.0 \mathrm{~g}$, and $15.0 \mathrm{~g})$ von Frey hairs (Stoelting Co., Wood Dale, Illinois, USA) by the up-down method as described previously [17, 18]. Briefly, the rats were accommodated in the testing chambers for a period of $30 \mathrm{~min}$ prior to the testing. A series of von Frey hairs with logarithmically incremental stiffness was applied to the periorbital region of the face and middle of the plantar surface of the front paw for 6$8 \mathrm{~s}$ at intervals of $30 \mathrm{~s}$ between consecutive stimuli. 
Quick withdrawal or licking of front paw in response to the stimulus or scratching of the periorbital region in response to the stimulus was considered as a positive response. The tactile thresholds to the stimuli of von Frey hairs were analyzed at baseline, 0.5, 1, 2, 3, and $4 \mathrm{~h}$ after NTG or NS injection by experimenters who were blinded to each rat group.

\section{Immunofluorescence staining}

Rats were anaesthetized with $10 \%$ chloral hydrate ( $3 \mathrm{ml} / \mathrm{kg}$, i.p) and then perfused transcardially with $0.9 \%$ saline at $4{ }^{\circ} \mathrm{C}$ followed by $4 \%$ paraformaldehyde in phosphate buffered saline (PBS $0.1 \mathrm{~mol} / \mathrm{L}, \mathrm{pH}$ 7.4). Regions from the medulla oblongata to the first cervical cord were immediately isolated, fixed in $4 \%$ paraformaldehyde, cryoprotected in $30 \%$ sucrose, frozen, and serially sectioned 1-5 mm from obex (10 $\mu \mathrm{m}$-thick transverse sections) on a cryostat (CM1900, Leica, Heidelberg, Germany). Sections were incubated with primary antibody against Nrf2 (rabbit polyclonal antibody, dilution 1:200, Abcam, UK), neuronal nuclei NeuN (mouse monoclonal antibody, dilution 1:500, Millipore, USA), c-Fos (rabbit monoclonal antibody, dilution 1:200, Abcam, UK), and nNOS (rabbit monoclonal antibody, dilution 1:200, CST, USA). After rinsing in PBS $(0.01 \mathrm{~mol} / \mathrm{L}, \mathrm{pH} 7.4)$, sections were incubated for $1 \mathrm{~h}$ at $25{ }^{\circ} \mathrm{C}$ with secondary antibody, Alexa Fluor 488conjugated anti-rabbit IgG (1:200, Jackson, USA) or Dylight 549-conjugated anti-mouse IgG (1:200, Jackson, USA). Sections were mounted in fluorescent mounting medium (R\&D systems, Minneapolis, MN, USA), and signals were detected using a fluorescence microscope (BX51, Olympus, Japan). Negative control sections were incubated with PBS instead of primary antibodies and they showed no positive signals. The number of c-Fos- and nNOS-immunoreactive neurons in TNC confined to a $400 \times 300 \mu \mathrm{m}$ square was determined using Image J software (version 1.4.3.67, NIH). Data from 10 regions sampled from each section (10 sections per rat) were averaged and presented as number per $1.2 \times 10^{5} \mu \mathrm{m}^{2}$ in TNC.

\section{Immunoblotting}

After anaesthetizing as described above, the TNC was rapidly dissected, 1-5 $\mathrm{mm}$ from obex, homogenized in tissue lysates (Pierce, Rockford, IL, USA) with protease inhibitor cocktail (Merck, Darmstadt, Germany), and the protein concentration was determined using BCA reagent (Pierce, Rockford, IL, USA). Equal amounts of protein extracts were separated electrophoretically on $10 \%$ sodium dodecyl sulfate-polyacrylamide gels and transferred onto polyvinylidene difluoride membranes (Millipore, Temecula, CA, USA). After blocking with $5 \%$ skimmed milk for $1 \mathrm{~h}$, the membranes were incubated with primary antibody against Nrf2 (rabbit polyclonal antibody, dilution 1:1000, Abcam, UK), HO1 (rabbit monoclonal antibody, dilution 1:1000, Abcam, UK), NQO1 (rabbit polyclonal antibody, dilution 1:1000, Abcam, UK), c-Fos (rabbit monoclonal antibody, dilution 1:500, Abcam, UK), nNOS (rabbit monoclonal antibody, dilution 1:1000, CST, USA), $\beta$-actin (mouse monoclonal antibody, dilution 1:5000, Sigma, USA), and fibrillarin (mouse monoclonal antibody, dilution 1:4000, Abcam, UK). For the negative control, rabbit primary antibody was replaced by normal serum. This was followed by adding horseradish peroxidase-conjugated anti-mouse or anti-rabbit secondary antibody (dilution 1:5000, Jackson, USA). An enhanced chemiluminescence kit (Millipore, Temecula, USA) was used for the visualization of the bands. Densitometric analysis was performed using Image J software.

\section{Statistical analysis}

All data are expressed as the mean \pm SD. Statistical analyses were performed using IBM SPSS version 17.0 (SPSS Inc., Chicago, IL, USA). Data at different time points were analyzed using a two-way analysis of variance (ANOVA) followed by Bonferroni post-hoc-test. Other data were analyzed using a one-way ANOVA followed by Bonferroni post-hoc-test. $P$ value $<0.05$ was considered as statistically significant.

\section{Results}

NTG altered the antioxidant system in TNC

To investigate the changes in the antioxidant system in rats treated with NTG, we analyzed Nrf2 expression in the nuclear and total cell fractions of TNC from rat models (Fig. 1). The subcutaneous administration of NTG $(10 \mathrm{mg} / \mathrm{kg})$ significantly increased Nrf2 levels in the total cell and nuclear fractions. This increase began within $0.5 \mathrm{~h}$ and persisted for $4 \mathrm{~h}$ after NTG injection. The control group with NS injection showed no statistical difference at the different time points. Moreover, immunofluorescence analysis (Fig. 2) showed that Nrf2 was located only in the neuronal cytoplasm of control group. Whereas, both nucleus and cytoplasm of neurons in the NTG group shared an obvious Nrf2 existence. We further analyzed the protein levels of two typical Nrf2regulated phase II enzymes, HO1 and NQO1, in TNC of the rat models (Fig. 3). The expression of these two proteins also increased within 0.5 or $1 \mathrm{~h}$, and persisted for $4 \mathrm{~h}$ after NTG exposure.

\section{Sulforaphane increased the expression of Nrf2 and the downstream proteins}

To determine the possible mechanism of Nrf2 effect, we used sulforaphane, a small-molecule inducer of this factor, to regulate the Nrf2/ARE pathway in this study. We performed immunoblotting to analyze the nuclear Nrf2, HO1, and NQO1 expressions. As shown in Fig. 4, 


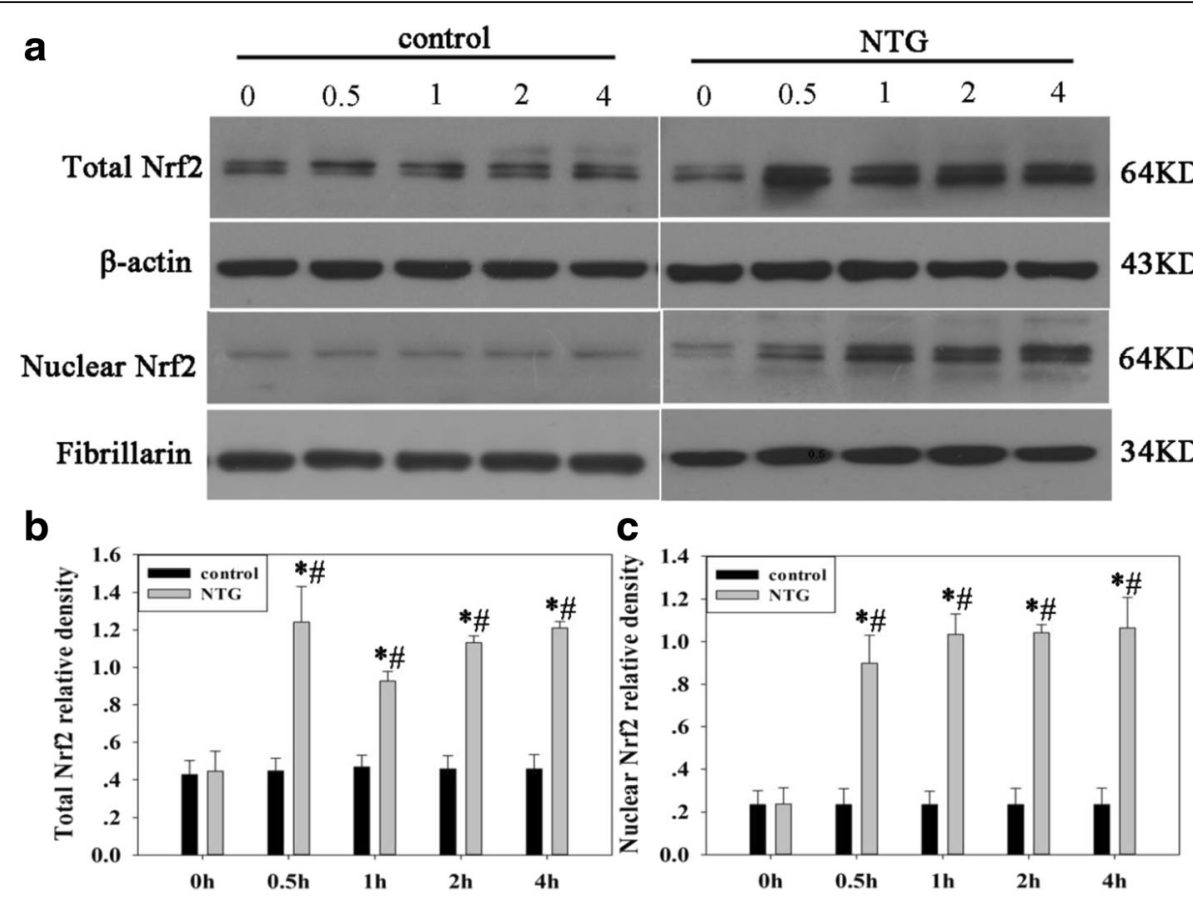

Fig. 1 Effect of NTG injection on Nrf2 protein levels in the total and nuclear fractions of rat TNC a Representative immunoblots of TNC lysates. Total Nrf2 levels $\mathbf{b}$ and nuclear Nrf2 levels $\mathbf{c}$ were elevated as early as $0.5 \mathrm{~h}$ and persisted for $4 \mathrm{~h}$ after NTG injection. $\beta$-actin was used as a loading control for total Nrf2. Fibrillarin was used to assess the purity of the nuclear fraction. Data are presented as relative density units normalized to $\beta$-actin or Fibrillarin, and expressed as mean \pm SD $(* P<0.01$ vs the control group, $\# P<0.01$ vs NTG 0 h group, $n=6$ per group)
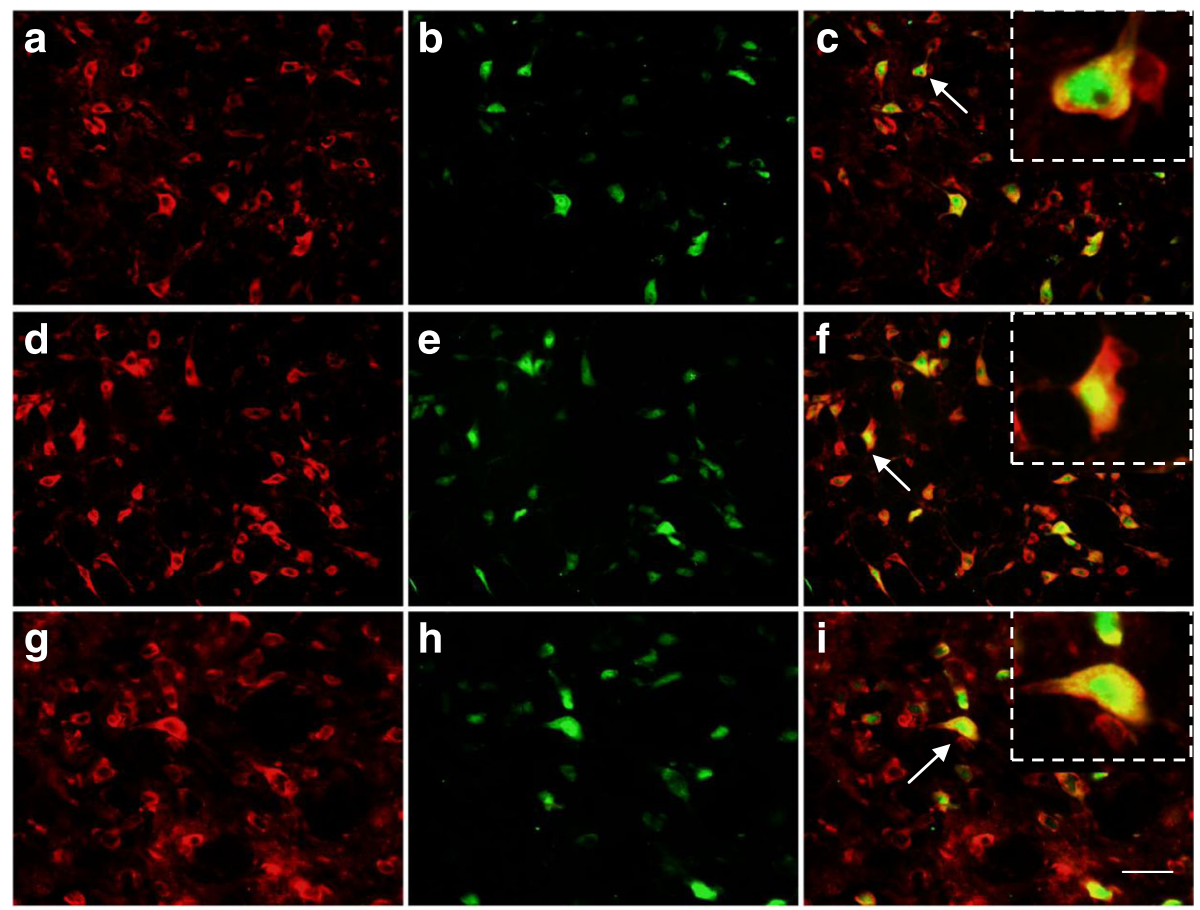

Fig. 2 Co-localization images of Nrf2 with NeuN in TNC. Red indicates Nrf2 immunoreactivity, green indicates NeuN immunoreactivity, and yellow indicates merged signal. In control group a-c of the TNC, Nrf2 is present mainly in the cytoplasm (shown by arrows). In the NTG $2 \mathrm{~h}$ group $\mathbf{d}$-f and NTG $4 \mathrm{~h}$ group $\mathbf{g}-\mathbf{i}$, Nrf2 staining was observed both in the cytoplasm and in the nucleus (shown by arrows). Bar $=50 \mu \mathrm{m}$. The arrows indicate cells shown in the top right corner of images $\mathbf{c}, \mathbf{f}, \mathbf{i}$ at about 10 times magnification 


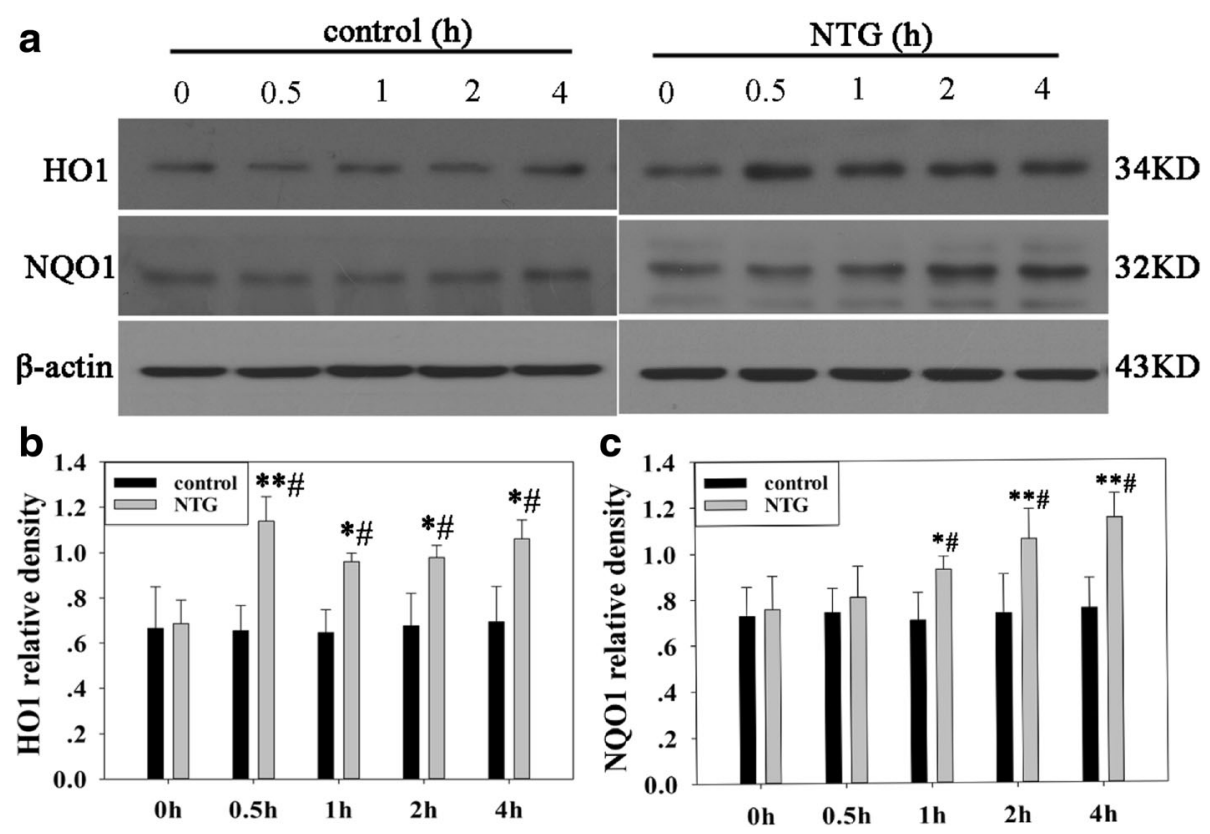

Fig. 3 Effect of NTG injection on the levels of Nrf2 downstream proteins HO1 and NQO1 in rat TNC. a Representative immunoblots of TNC. Compared to the NS group or NTG 0 h group, HO1 levels were elevated at $0.5 \mathrm{~h}, 1 \mathrm{~h}, 2 \mathrm{~h}$, and $4 \mathrm{~h}$ after NTG injection $\mathbf{b}$ and NQO1 levels were elevated at 1 h, 2 h, and 4 h after NTG injection c. $\beta$-actin was used as the loading control. Data are presented as relative density units normalized to $\beta$-actin, and expressed as mean \pm SD. ( ${ }^{*} P<0.05$ vs the control group, ${ }^{* *} P<0.01$ vs the control group, $\# P<0.01$ vs NTG 0 h group, $n=6$ per group)

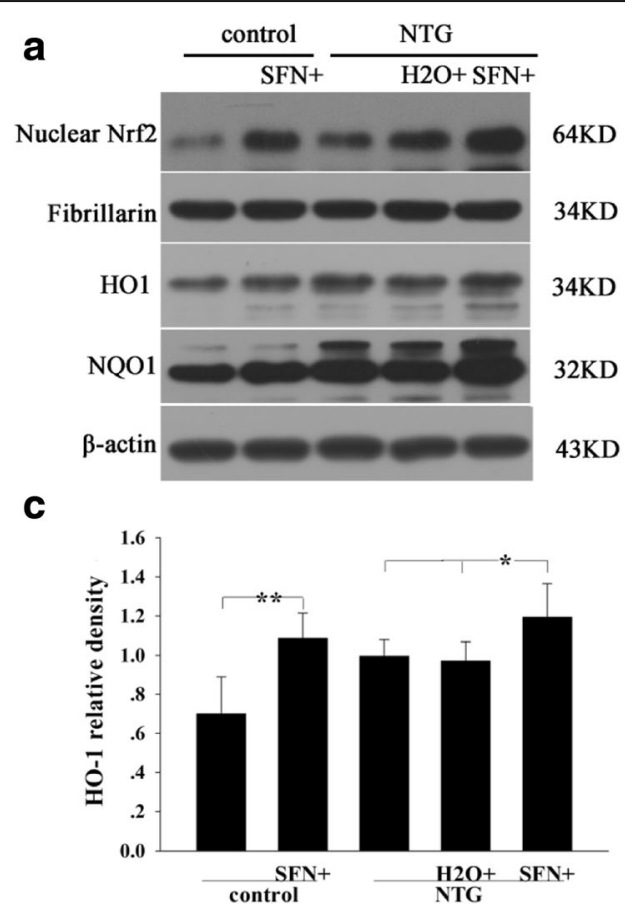

b

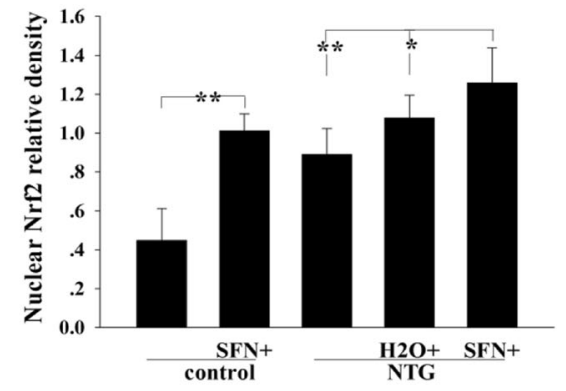

d

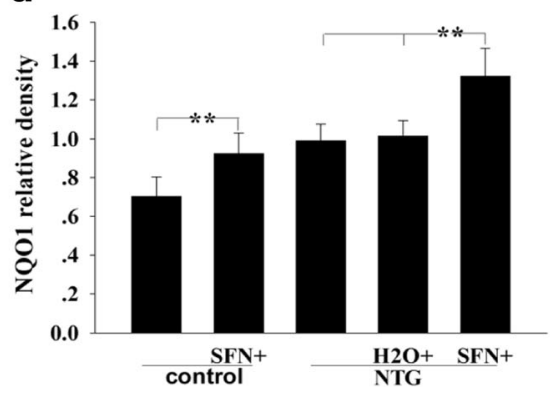

Fig. 4 Effect of sulforaphane (SFN) on the levels of Nrf2 and downstream proteins HO1 and NQO1 in rat TNC $4 \mathrm{~h}$ after NTG injection. a Representative immunoblots of TNC. Nuclear Nrf2 b, HO1 c and NQO1 d levels were significantly increased in the SFN plus NTG group compared to those in the $\mathrm{H}_{2} \mathrm{O}$ plus NTG group. Moreover, these protein expressions were increased in the SFN plus control group compared to those in the control group $\mathbf{b}, \mathbf{c}, \mathbf{d}$. Data are presented as the mean \pm SD. ( ${ }^{*} P<0.05,{ }^{* *} P<0.01, n=6$ per group) 
sulforaphane treatment significantly increased nuclear Nrf2 level in NTG-treated rats (Fig. 4b). Moreover, it resulted in a significant increase in $\mathrm{HO} 1$ and NQO1 levels in these animals, compared to those in the control samples (Fig. 4c and d). Meanwhile, these protein expression levels were also increased in the sulforaphane plus control group compared to those in the control group.

\section{Sulforaphane pretreatment inhibited NTG-induced TGVS activation}

Increased neuronal nitric oxide synthase (nNOS) and pro-oncogene c-Fos expressions in TNC are considered as the markers of TGVS activation $[19,20]$. To check whether Nrf2 was involved in NTG-induced TGVS activation, we measured the expression levels of nNOS and c-Fos in NTG-treated samples at $4 \mathrm{~h}$ after sulforaphane pretreatment. As shown in Fig. 5, sulforaphane significantly reversed NTG-induced increase in nNOS and c-Fos expression. It also significantly reduced the number of nNOS- and c-Fos-immunoreactive neurons in TNC (Fig. 6).

\section{Sulforaphane pretreatment alleviated the NTG-induced cutaneous hyperalgesia}

We further investigated the efficacy of sulforaphane pretreatment on tactile sensitivity with calibrated von Frey hairs. We observed that subcutaneous administration of NTG significantly reduced the periorbital sensory and front paw withdrawal thresholds compared to that seen in the control group, which began $0.5 \mathrm{~h}$ after NTG injection (Fig. 7). Withdrawal thresholds of the front paw were significantly elevated in sulforaphanepretreatment rats from 0.5-4 $\mathrm{h}$ after NTG injection (Fig. 7a). For periorbital tactile threshold analysis, an obvious increase was observed in the sulforaphane group from 1-4 h after NTG injection, as compared to the vehicle group (Fig. 7b).

\section{Discussion}

Our study showed that the Nrf2/ARE signaling pathway in TNC was activated during NTG-induced migraine in rats. Sulforaphane pretreatment enhanced Nrf2 activation, increased the expression of $\mathrm{HO} 1$ and NQO1, decreased the expression of nNOS and c-Fos, and alleviated the NTG-induced hyperalgesia. These results indicated that oxidative stress was involved in NTGinduced hyperalgesia. Antioxidants may alleviate hyperalgesia via the suppression of TGVS activation. This study showed for the first time that sulforaphane, a natural Nrf2 activator compound, plays a protective role in NTG-induced hyperalgesia.

Under normal conditions, Nrf2 existence remains in the cytosol. Oxidative stressors can cause Nrf2 to translocate to the nucleus, thereby activating the Nrf2 pathway [21]. In this study, we observed that subcutaneous administration of NTG significantly increased nuclear

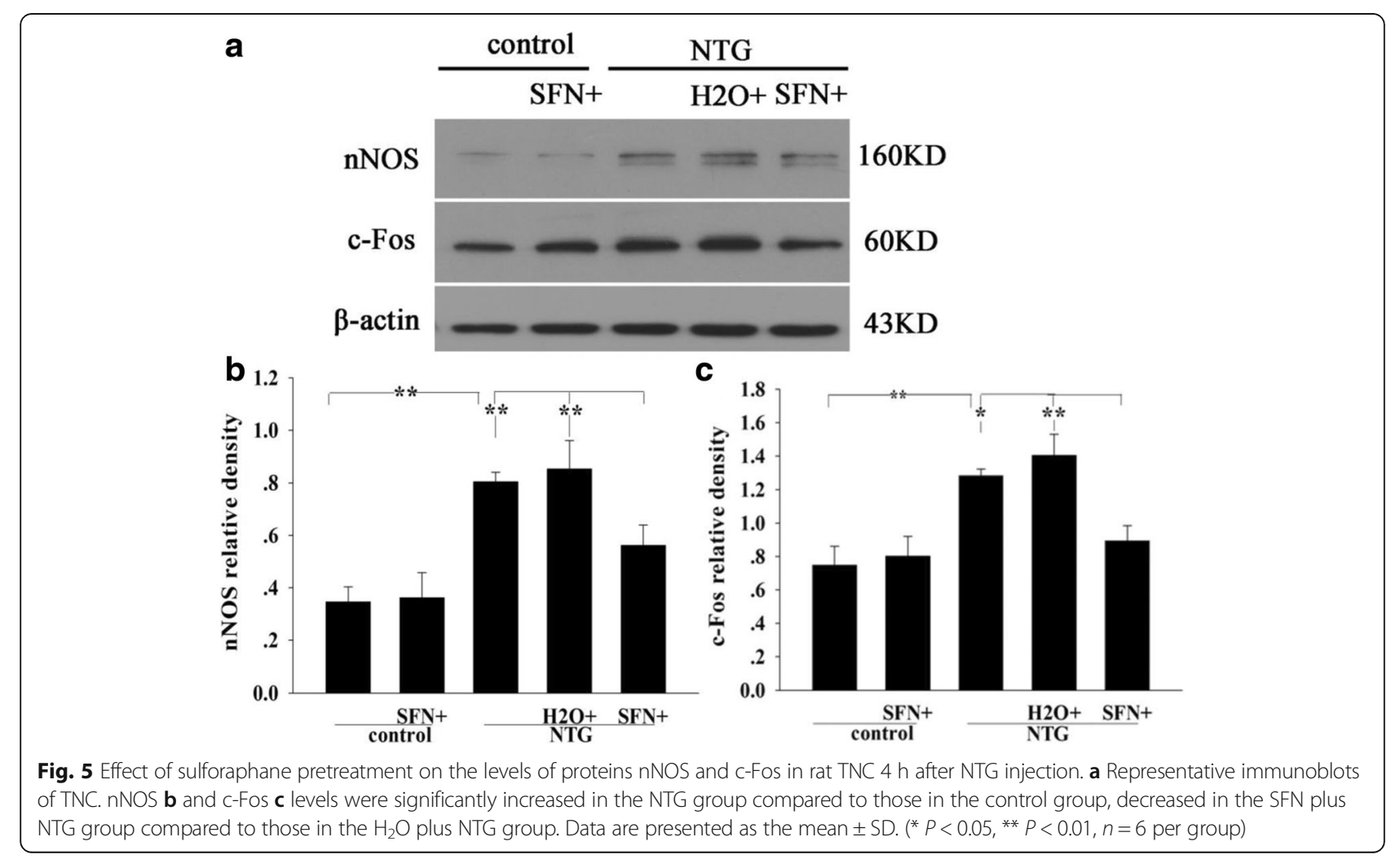



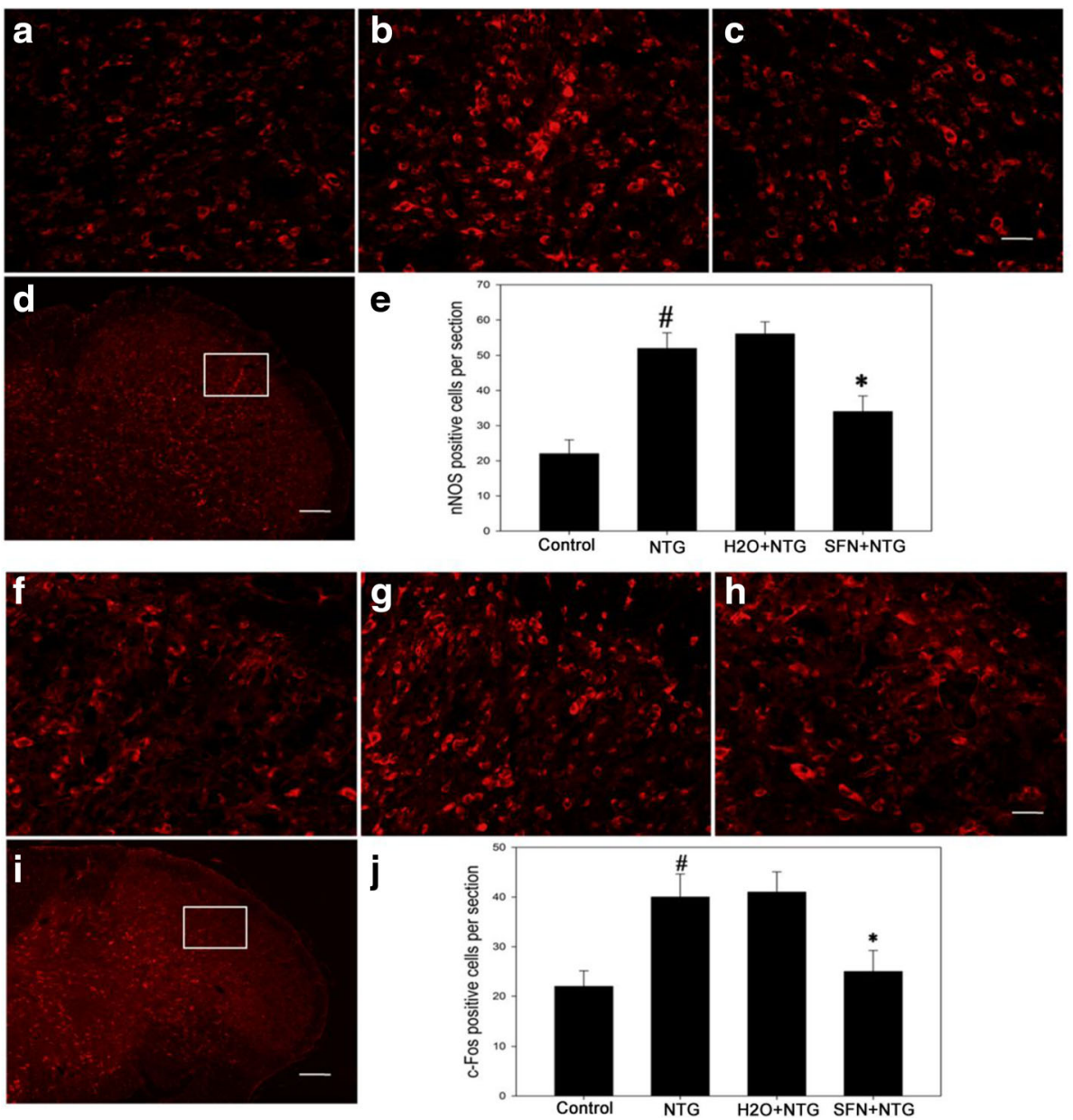

Fig. 6 Effect of sulforaphane pretreatment on the number of nNOS- and c-Fos-immunoreactive cells in rat TNC $4 \mathrm{~h}$ after NTG injection. Panels a-d correspond to nNOS immunofluorescence staining, panels f-i correspond to c-Fos immunofluorescence staining. a,f control group. $\mathbf{b}, \mathbf{g}$ NTG group. $\mathbf{c}, \mathbf{h}$ SFN + NTG group. $\mathbf{d}, \mathbf{i}$ the white rectangle frame indicates the representative anatomical site observed. Quantitative analysis showed that nNOS-immunoreactive $\mathbf{e}$ and c-Fos-immunoreactive cells $\mathbf{j}$ were all increased in the NTG group compared to those in the control group, but decreased in the SFN plus NTG group compared to those in the $\mathrm{H}_{2} \mathrm{O}$ plus NTG group. ( ${ }^{*} P<0.05$ for SFN $+\mathrm{NTG}$ vs $\mathrm{H}_{2} \mathrm{O}+\mathrm{NTG}$ group, \# $P<0.05$ for NTG vs control group, $n=6$ per group) (a, b, c, f, g, h: Bar $=50 \mu \mathrm{m} ; \mathrm{d}$, i: Bar $=200 \mu \mathrm{m})$

Nrf2 expression in rat TNC. The levels of the two typical Nrf2-regulated phase II enzymes, HO1 and NQO1, were also increased. These data indicate that NTG induces oxidative stress, which contributes to the activation of Nrf2/ARE pathway. Moreover, NTG-induced oxidative stress has been proved to be involved in migraine pathogenesis $[22,23]$. Thus, we believe that the Nrf2/ARE pathway is an endogenous adaptive compensatory factor in migraine. Similar to the situation in NTG-treated changes, Nrf2/ARE pathway is also activated in ischemic stroke, traumatic brain injury, and subarachnoid hemorrhage, and it shows compensatory adaptation [24-26]. Despite these observations, the mechanism of Nrf2/ARE pathway activation needs to be investigated further. NO induces Keap1 disulfide formation, Keap1 S-nitrosylation, or Keap1 S-guanylation. It can also induce oxidative or nitrosative stress which possibly induce the following activation of Nrf2, directly or indirectly through CRM1 or PI3K/PKC signaling pathway [27]. We also found that the total cellular Nrf2 expression was elevated. Consistent with the findings of the present study, inorganic arsenic induced an increase in Nrf2 protein by enhancing Nrf2 transcription [28]. We suggest that NTG may promote Nrf2 transcription, thereby increasing the Nrf2 protein level.

Activation of the Nrf2/ARE pathway is critical for neuroprotection [14, 29]. As an activator of Nrf2 pathway, sulforaphane is well known for its antioxidant and detoxification effects by inducing phase II genes [13]. We found in this study that sulforaphane activated Nrf2, upregulated downstream $\mathrm{HO} 1$ and NQO1, suppressed TGVS activation, and ameliorated the decrease of tactile thresholds in NTG-induced rats. These findings indicated that sulforaphane was probably involved in anti- 


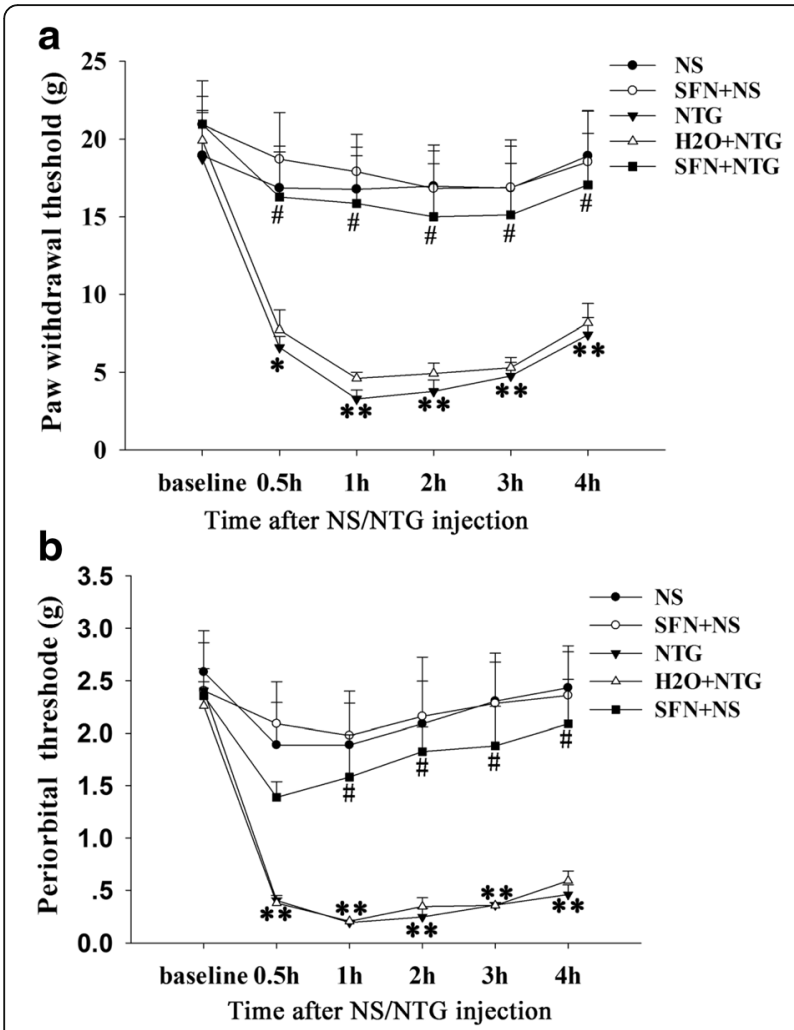

Fig. 7 Effect of sulforaphane pretreatment on front paw withdrawal and periorbital thresholds in NTG-induced rats. a The front paw withdrawal thresholds significantly decreased as early as $0.5 \mathrm{~h}$ and persisted for $4 \mathrm{~h}$ in the NTG group compared to those in the control group, whereas the thresholds significantly increased as early as $0.5 \mathrm{~h}$ and persisted for $4 \mathrm{~h}$ in the SFN plus NTG group compared to those in the $\mathrm{H}_{2} \mathrm{O}$ plus NTG group. b The periorbital withdrawal thresholds also significantly decreased as early as $0.5 \mathrm{~h}$ and persisted for $4 \mathrm{~h}$ in the NTG group, while the thresholds significantly increased as early as $1 \mathrm{~h}$ and persisted for $4 \mathrm{~h}$ in the SFN plus NTG group compared to those in the $\mathrm{H}_{2} \mathrm{O}$ plus NTG group. $\left({ }^{*} P<0.05\right.$, ${ }^{*} P<0.01$ for NTG vs control group, ${ }^{\#} P<0.05$ for SFN + NTG vs $\mathrm{H}_{2} \mathrm{O}+\mathrm{NTG}$ group. $n=6$ per group)

hyperalgesia through the anti-oxidative stress Nrf2/ARE pathway. In addition, previous studies have shown that Nrf2-/- cells exhibit increased nNOS and c-Fos expression and oxidative damage [30,31]. These data were similar to our findings of the suppressive effects of Nrf2 on nNOS and c-Fos. It is believed that activation of Nrf2 would inhibit TGVS ability by down-regulating nNOS and c-Fos expression. The above mechanisms may partly account for the efficacy of sulforaphane in migraine treatment. Moreover, anti-hyperalgesia efficacy of sulforaphane suggests that this compound reduces the production of proinflammatory cytokines and inhibits microglia activation [32-34]. These anti-inflammatory effects of sulforaphane may also contribute to its role in migraine treatment.

The present study shows that sulforaphane plays a therapeutic role in migraine solely via TNC neuronal activation. In future studies, Nrf2 gene knockout rats could be used to investigate the protective effect of sulforaphane. The underlying mechanism of sulforaphane action on migraine needs further investigation.

\section{Conclusions}

In summary, our study initially demonstrated a critical role of the Nrf2/ARE signaling pathway in NTG-induced hyperalgesia rats. Our findings indicated that an Nrf2 activator, sulforaphane, inhibited the trigeminovascular system activation and prevented the induction of hyperalgesia, which provides a novel insight into the potential application of antioxidants as novel candidates in drug development for migraine.

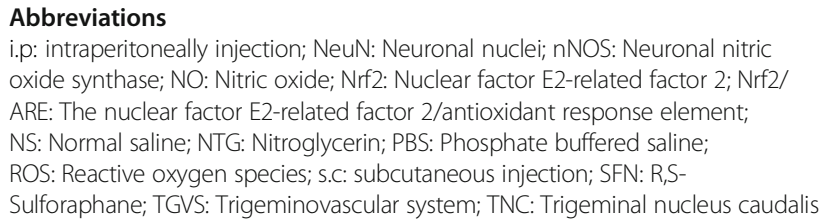

\section{Acknowledgements}

This study was supported by grants from the National Natural Science Fundation of China (No. 81500965, 81171101). Science and Technology Program of Guangzhou (No. 201508020026), Guangdong Provincial Key Laboratory for Diagnosis and Treatment of Major Neurological Diseases (2014B030301035) and Guangzhou National Key Discipline and National Key Clinical Department.

\section{Availability of data and materials}

The datasets supporting the conclusions of this article are included within the article.

\section{Authors' contributions}

YF, WD participated in the whole design of this study. WD, XS and HL performed the animal model preparation, immunofluorescence, western blot and data analysis. JL, ZL participated in Behavior test and data analysis; WD, XS and $\mathrm{HZ}$ wrote the draft and revised the whole manuscript. All authors read and approved the final version of the manuscript.

\section{Competing interests}

The authors declare that they have no competing interests.

\section{Consent for publication}

Not applicable.

\section{Ethics approval}

All animal experiments in this study were carried out in accordance with the Institutional Animal Ethical Committee of Sun Yat-sen University and were approved by the Institutional Animal Care and Use Committee guidelines (Publication No.80-23, revised in 1996)

\section{Author details}

1Department of Neurology, Shaanxi Provincial People's Hospital, The Third Affiliated Hospital of Xi'an Jiaotong University, Shaanxi 710068, China. ${ }^{2}$ Department of Neurology, The First Affiliated Hospital, Yijishan Hospital of Wannan Medical College, Anhui 241001, China. ${ }^{3}$ Guangdong Key Laboratory for Diagnosis and Treatment of Major Neurological Diseases, Department of Neurology, National Key Clinical Department and Key Discipline of Neurology, The First Affiliated Hospital, Sun Yat-sen University, Guangdong 510080, China.

Received: 9 August 2016 Accepted: 19 October 2016

Published online: 24 October 2016 


\section{References}

1. Lipton RB, Bigal ME, Ashina S, Burstein R, Silberstein S, Reed ML, Serrano D, Stewart WF (2008) Cutaneous allodynia in the migraine population. Ann Neurol 63(2):148-158. doi:10.1002/ana.21211

2. Louter MA, Bosker JE, van Oosterhout WP, van Zwet EW, Zitman FG, Ferrari MD, Terwindt GM (2013) Cutaneous allodynia as a predictor of migraine chronification. Brain 136(Pt 11):3489-3496. doi:10.1093/brain/awt251

3. Bates EA, Nikai T, Brennan KC, Fu YH, Charles AC, Basbaum Al, Ptacek $\sqcup$, Ahn AH (2010) Sumatriptan alleviates nitroglycerin-induced mechanical and thermal allodynia in mice. Cephalalgia 30(2):170-178. doi:10.1111/j.1468-2982.2009.01864.x

4. Greco R, Mangione AS, Siani F, Blandini F, Vairetti M, Nappi G, Sandrini G, Buzzi MG, Tassorelli C (2014) Effects of CGRP receptor antagonism in nitroglycerin-induced hyperalgesia. Cephalalgia 34(8):594-604 doi:10.1177/0333102413517776

5. Tassorelli C, Greco R, Sandrini G, Nappi G (2003) Central components of the analgesic/antihyperalgesic effect of nimesulide: studies in animal models of pain and hyperalgesia. Drugs 63(Suppl 1):9-22

6. Iversen HK, Olesen J, Tfelt-Hansen P (1989) Intravenous nitroglycerin as an experimental model of vascular headache. Basic characteristics. Pain 38(1):17-24

7. Christiansen I, Daugaard D, Lykke Thomsen L, Olesen J (2000) Glyceryl trinitrate induced headache in migraineurs - relation to attack frequency. Eur J Neurol 7(4):405-411

8. de Tommaso M, Libro G, Guido M, Difruscolo O, Losito L, Sardaro M, Cerbo R (2004) Nitroglycerin induces migraine headache and central sensitization phenomena in patients with migraine without aura: a study of laser evoked potentials. Neurosci Lett 363(3):272-275. doi:10.1016/j.neulet.2004.04.029

9. Lee I, Kim HK, Kim JH, Chung K, Chung JM (2007) The role of reactive oxygen species in capsaicin-induced mechanical hyperalgesia and in the activities of dorsal horn neurons. Pain 133(1-3):9-17. doi:10.1016/j.pain.2007.01.035

10. Nishio N, Taniguchi W, Sugimura YK, Takiguchi N, Yamanaka M, Kiyoyuki Y, Yamada H, Miyazaki N, Yoshida M, Nakatsuka T (2013) Reactive oxygen species enhance excitatory synaptic transmission in rat spinal dorsal horn neurons by activating TRPA1 and TRPV1 channels. Neuroscience 247:201-212. doi:10.1016/j.neuroscience.2013.05.023

11. Gao X, Kim HK, Chung JM, Chung K (2007) Reactive oxygen species (ROS) are involved in enhancement of NMDA-receptor phosphorylation in animal models of pain. Pain 131(3):262-271. doi:10.1016/j.pain.2007.01.011

12. Kim HK, Park SK, Zhou JL, Taglialatela G, Chung K, Coggeshall RE, Chung JM (2004) Reactive oxygen species (ROS) play an important role in a rat model of neuropathic pain. Pain 111(1-2):116-124. doi:10.1016/j.pain.2004.06.008

13. Lee JM, Johnson JA (2004) An important role of Nrf2-ARE pathway in the cellular defense mechanism. J Biochem Mol Biol 37(2):139-143

14. Zhang M, An C, Gao Y, Leak RK, Chen J, Zhang F (2013) Emerging roles of Nrf2 and phase II antioxidant enzymes in neuroprotection. Prog Neurobiol 100:30-47. doi:10.1016/j.pneurobio.2012.09.003

15. Ping Z, Liu W, Kang Z, Cai J, Wang Q, Cheng N, Wang S, Wang S, Zhang JH, Sun X (2010) Sulforaphane protects brains against hypoxic-ischemic injury through induction of Nrf2-dependent phase 2 enzyme. Brain Res 1343:178-185 doi:10.1016/i.brainres.2010.04.036

16. Wang $X$, de Rivero Vaccari JP, Wang H, Diaz P, German R, Marcillo AE, Keane RW (2012) Activation of the nuclear factor E2-related factor 2/antioxidant response element pathway is neuroprotective after spinal cord injury. J Neurotrauma 29(5):936-945. doi:10.1089/neu.2011.1922

17. Chaplan SR, Bach FW, Pogrel JW, Chung JM, Yaksh TL (1994) Quantitative assessment of tactile allodynia in the rat paw. J Neurosci Methods 53(1):55-63

18. De Felice M, Ossipov MH, Wang R, Lai J, Chichorro J, Meng I, Dodick DW, Vanderah TW, Dussor G, Porreca F (2010) Triptan-induced latent sensitization: a possible basis for medication overuse headache. Ann Neurol 67(3):325-337. doi:10.1002/ana.21897

19. Pardutz A, Krizbai I, Multon S, Vecsei L, Schoenen J (2000) Systemic nitroglycerin increases nNOS levels in rat trigeminal nucleus caudalis. Neuroreport 11(14):3071-3075

20. Ramachandran R, Bhatt DK, Ploug KB, Olesen J, Jansen-Olesen I, Hay-Schmidt A, Gupta S (2012) A naturalistic glyceryl trinitrate infusion migraine model in the rat. Cephalalgia 32(1):73-84. doi:10.1177/0333102411430855

21. Kaspar JW, Niture SK, Jaiswal AK (2009) Nrf2:INrf2 (Keap1) signaling in oxidative stress. Free Radic Biol Med 47(9):1304-1309. doi:10.1016/j.freeradbiomed. 2009.07.035

22. Gruber HJ, Bernecker $C$, Lechner A, Weiss S, Wallner-Blazek M, Meinitzer A Hobarth G, Renner W, Fauler G, Horejsi R, Fazekas F, Truschnig-Wilders M (2010) Increased nitric oxide stress is associated with migraine. Cephalalgia 30(4):486-492. doi:10.1111/j.1468-2982.2009.01964.x
23. Li Y, Zhang Q, Qi D, Zhang L, Yi L, Li Q, Zhang Z (2016) Valproate ameliorates nitroglycerin-induced migraine in trigeminal nucleus caudalis in rats through inhibition of NF-small ka, CyrillicB. J Headache Pain 17:49. doi:10.1186/s10194016-0631-z

24. Wang Z, Chen G, Zhu WW, Zhou D (2010) Activation of nuclear factorerythroid 2-related factor 2 (Nrf2) in the basilar artery after subarachnoid hemorrhage in rats. Ann Clin Lab Sci 40(3):233-239

25. Yan W, Wang HD, Feng XM, Ding YS, Jin W, Tang K (2009) The expression of NF-E2-related factor 2 in the rat brain after traumatic brain injury. J Trauma 66(5):1431-1435. doi:10.1097/TA.0b013e318180f5c7

26. Tanaka N, Ikeda Y, Ohta Y, Deguchi K, Tian F, Shang J, Matsuura T, Abe K (2011) Expression of Keap1-Nrf2 system and antioxidative proteins in mouse brain after transient middle cerebral artery occlusion. Brain Res 1370:246-253. doi:10.1016/j.brainres.2010.11.010

27. Liu W, Wang D, Liu K, Sun X (2012) Nrf2 as a converging node for cellular signaling pathways of gasotransmitters. Med Hypotheses 79(3):308-310. doi:10.1016/j.mehy.2012.05.016

28. Zhang Y, Duan X, Li J, Zhao S, Li W, Zhao L, Li W, Nie H, Sun G, Li B (2016) Inorganic Arsenic Induces NRF2-Regulated Antioxidant Defenses in Both Cerebral Cortex and Hippocampus in Vivo. Neurochem Res 41(8):2119-2128. doi:10.1007/s11064-016-1927-8

29. Takagi T, Kitashoji A, Iwawaki T, Tsuruma K, Shimazawa M, Yoshimura S, Iwama T, Hara H (2014) Temporal activation of Nrf2 in the penumbra and Nrf2 activator-mediated neuroprotection in ischemia-reperfusion injury. Free Radic Biol Med 72:124-133. doi:10.1016/j.freeradbiomed.2014.04.009

30. Hyeon $S$, Lee $H$, Yang $Y$, Jeong W (2013) Nrf2 deficiency induces oxidative stress and promotes RANKL-induced osteoclast differentiation. Free Radic Biol Med 65:789-799. doi:10.1016/j.freeradbiomed.2013.08.005

31. Minelli A, Conte C, Grottelli S, Bellezza I, Cacciatore I, Bolanos JP (2009) Cyclo(His-Pro) promotes cytoprotection by activating Nrf2-mediated upregulation of antioxidant defence. J Cell Mol Med 13(6):1149-1161. doi:10.1111/j.1582-4934.2008.00326.x

32. Kim D, You B, Jo EK, Han SK, Simon MI, Lee SJ (2010) NADPH oxidase 2-derived reactive oxygen species in spinal cord microglia contribute to peripheral nerve injury-induced neuropathic pain. Proc Natl Acad Sci U S A 107(33):14851-14856. doi:10.1073/pnas.1009926107

33. Negi G, Kumar A, Sharma SS (2011) Nrf2 and NF-kappaB modulation by sulforaphane counteracts multiple manifestations of diabetic neuropathy in rats and high glucose-induced changes. Curr Neurovasc Res 8(4):294-304

34. Sun CC, Li SJ, Yang CL, Xue RL, Xi YY, Wang L, Zhao QL, Li DJ (2015) Sulforaphane Attenuates Muscle Inflammation in Dystrophin-deficient mdx Mice via NF-E2 related Factor 2 (Nrf2)-mediated Inhibition of NF-kappaB Signaling Pathway. J Biol Chem 290(29):17784-17795. doi:10.1074/jbc.M115.655019

\section{Submit your manuscript to a SpringerOpen ${ }^{\circ}$ journal and benefit from:}

- Convenient online submission

- Rigorous peer review

- Immediate publication on acceptance

Open access: articles freely available online

- High visibility within the field

- Retaining the copyright to your article

Submit your next manuscript at $>$ springeropen.com 